\title{
NORMS OF POSITIVE DEFINITE TOEPLITZ MATRICES AND DETECTION OF ALMOST PERIODIC COMPONENTS IN RANDOM SIGNALS
}

\author{
VADYM ADAMYAN, JOSÉ LUIS ISERTE, \\ IGOR M. TKACHENKO AND GUMERSINDO VERDÚ
}

Abstract. For positive definite Toeplitz matrices $Q_{N}=(b(j-k))_{j, k=0}^{N-1}$ generated by trigonometric moments $b(j)$ of a non-negative measure $d \sigma(\theta), \theta \in[-\pi, \pi]$, we note that the HilbertSchmidt norm $\left\|Q_{N}\right\|_{2}$ and the maximal eigenvalue $\lambda_{m}(N)$ satisfy the following relations

$$
\lim _{N \rightarrow \infty} \frac{1}{N^{2}}\left\|Q_{N}\right\|_{2}^{2}=\sum_{\alpha} \mathfrak{m}_{\alpha}^{2}, \quad \lim _{N \rightarrow \infty} \frac{1}{N} \lambda_{m}(N)=\max _{\alpha} \mathfrak{m}_{\alpha},
$$

where $\left\{\mathfrak{m}_{\alpha}\right\}$ is the set of jumps of $\sigma(\theta)$. Analogous relations hold for positive definite integral operators with difference kernels. The above relations are used in order to detect hidden almost periodic components in random signals.

Mathematics subject classification (2010): Primary 47B35; Secondary 15B05, 45E10, 60G10, 60 G35. Keywords and phrases: Positive definite Toeplitz matrices, stationary processes, positive integral operators with difference kernels, signal processing.

\section{REFERENCES}

[1] A. BötTCHeR AND S. GRUdSky, Spectral properties of banded Toeplitz matrices, SIAM, Philadelphia, 2005.

[2] A. BötTcher And B. Silbermann, Introduction to large truncated Toeplitz matrices, Springer, New York, 1999.

[3] Yu. A. Rozanov, Stationary random processes, Holden-Day, San Francisco, 1967.

[4] G. Verdú, D. Ginestar, J. L. Muñoz-Cobo, J. Navarro, M. J. Palomo, P. Lansaker, J. M. CONDE, M. ReCIO, E. SARTORI, Forsmark $1 \& 2$ Boiling Water Reactor Stability benchmark, Time Series Analysis Methods for Oscillation during BWR Operation, Final Report. Nuclear Energy Agency, Organization for Economic Co-Operation and Development, NEA/NSC/DOC/(2001).

[5] N. WIENER, The quadratic variation of a function and its Fourier coefficients, Massachusett's Journal of Math., 3 (1924), 72-94.

[6] N. WiEnER, The Fourier integral and certain of its applications, Cambridge Mathematical Library, Cambridge University Press, 1988.

[7] A. Zygmund, Trigonometric series, Vol. I, II, Cambridge Mathematical Library, Cambridge University Press, 2002. 